\title{
Real-Time Selection of Pulse Repetition Frequency (PRF) Set for a Triple 2-of-3 PRF Scheme
}

\author{
Tae-Hyung $\mathrm{Kim}^{1,{ }^{*}} \cdot$ Jae-Woong $\mathrm{Yi}^{2} \cdot$ Young-Jin Byun ${ }^{2}$
}

\begin{abstract}
A triple 2-of-3 pulse repetition frequency (PRF) scheme is presented for medium PRF pulsed-Doppler airborne radars, and a real-time method is developed that searches for optimal or sub-optimal PRF sets according to momentary battle situations. The effectiveness of the real-time search method of PRF sets is demonstrated by the experimental results obtained using simulated data.
\end{abstract}

Key Words: Clutter, MPRF Pulsed-Doppler Radar, Real-Time PRF Scheduling, Selection of PRF Sets, Visibility of PRF Sets.

\section{Introduction}

A medium pulse repetition frequency (PRF) pulsed-Doppler radar commonly resolves the ambiguities of target returns by using an $M$-of- $N$ scheme [1]. $M$-of- $N$ schemes require the selection of suitable combinations of PRFs to resolve these ambiguities as well as to minimize blind regions by clutter and eclipsing losses in range and velocity regions of interest, to avoid blind velocities, and to reduce ghosting problems [2]. The optimal set of $N$ PRFs for an $M$-of- $N$ scheme changes according to the battle conditions (altitude, range, velocity, antenna scan positions, and etc.). Therefore, the operating PRF sets used in real time must be reconfigured according to the ever-changing conditions [2]. A common choice for $M$ and $N$ is 3-of-8; however, because of the many combinations of PRF sets, the 3-of-8 scheme requires costly microprocessors to search for the optimal or sub-optimal PRF schedules in real time. On the other hand, since a 2-of-3 scheme is based on relatively few combinations of PRF sets, the operating PRF sets can be selected in real time without the use of costly microprocessors.

The problem is that a 2-of-3 PRF schedule has serious problems of ghosting. Nevertheless, ghosting problems can be mitigated by circular switching of the operating set among the multiple PRF sets at an instant of target detection [3]. Therefore, in this letter, we propose the use of a triple 2-of-3 PRF schedule for real-time searching of
PRF sets and elimination of ghost targets. We describe a real-time search method for the optimal (and sub-optimal) 2-of-3 PRF schedules and the application of the triple 2of-3 PRF schedule are described in medium PRF pulsed-Doppler airborne radars.

\section{Selection of PRF Set}

A method is proposed for selecting the 2-of-3 PRF schedule. Constraints affecting the selection of the PRF set are decodability, ghosting, minimization of blind regions, and bounds of available PRFs (the number $N$ of available PRFs), etc. The decodability constraints described in [1] can be used. Decodability depends on maximum detection range and Doppler, independent of battle conditions. Therefore, PRF sets are selected as follows: 1) the PRF sets that satisfy decodability constraints are preselected from ${ }_{N} \mathrm{C}_{3}$ PRF sets and stored; 2) these pre-selected PRF sets are prioritized according to the minimization constraints of the blind regions for each battle condition; and 3) three PRF sets are selected by considering these priorities. A PRF set having minimum blind regions is revealed by calculating the visibility in the range-Doppler region of interest (ROI). We calculated the visibility of a PRF set in the ROI by first defining and calculating the blindness margin for the mainlobe clutter (MLC), sidelobe clutter (SLC), and eclipse, which cause blindness. These blinding factors, which hide targets, repeat at PRF

Manuscript received March 28, 2013 ; Revised May 31, 2013 ; Accepted July 19, 2013. (ID No. 20130328-009J)

${ }^{1}$ ISR R\&D Lab., LIG Nex1 Co., Ltd., Yongin, Korea.

${ }^{2}$ Agency for Defense Development, Daejeon, Korea.

${ }^{*}$ Corresponding Author : Tae-Hyung Kim (e-mail : thkim07@gmail.com)

This is an Open-Access article distributed under the terms of the Creative Commons Attribution Non-Commercial License (http://creativecommons.org/licenses/ by-nc/3.0) which permits unrestricted non-commercial use, distribution, and reproduction in any medium, provided the original work is properly cited. 


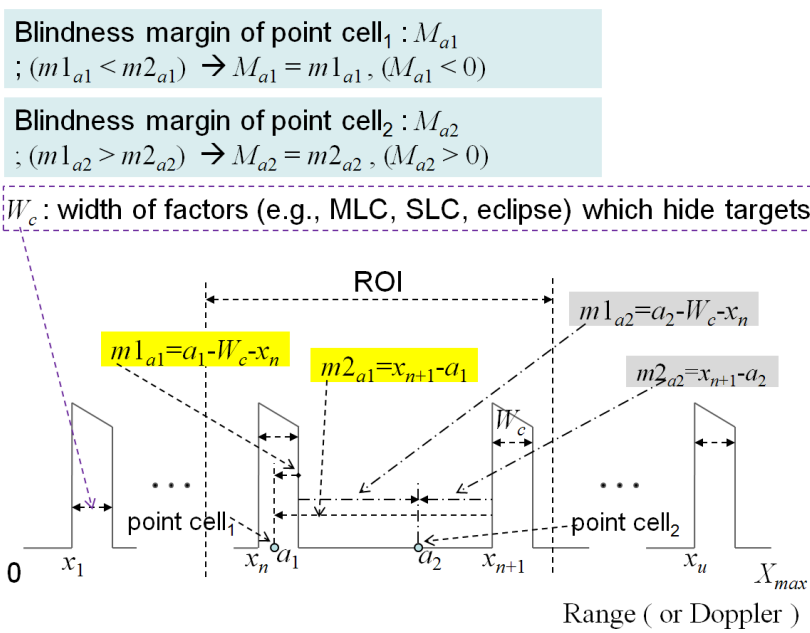

Fig. 1. Calculation of blindness margin for a point cell in region of interest (ROI). MLC=mainlobe clutter, SLC $=$ sidelobe clutter.

(or pulse repetition interval) intervals, as shown in Fig. 1. Placement of a target of a point cell $1_{1}$ within the width of blinding factors - in other words, when the blindness margin of a target is negative--renders the target invisible.

The widths of the blinding factors are as follows: the MLC width is the rejection notch bandwidth of MLC (MLC width is determined by flight speed, beamwidth of antenna, and etc.); the width for eclipse is the transmitted pulse width; and the SLC width is determined as shown in Fig. 2.

In Fig. 2, the SLC power at a range is calculated using a surface reflectivity model, flight altitude, etc., and the minimum target power in the ROI (for the worst case) is calculated considering a certain target. The SLC width is

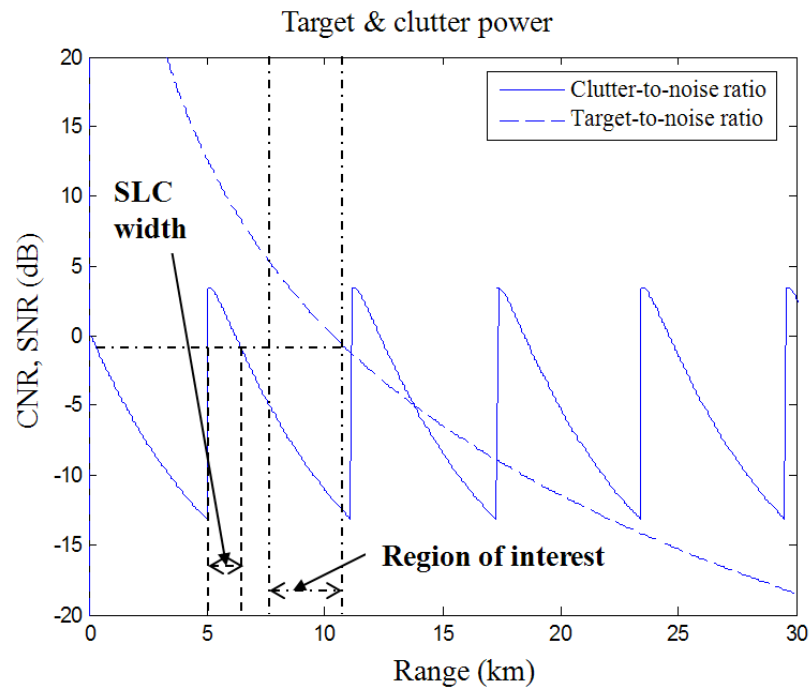

Fig. 2. Sidelobe clutter (SLC) and target power, and determination of SLC width in region of interest. CNR $=$ clutter-to-noise ratio, $\mathrm{SNR}=$ signal-to-noise ratio.
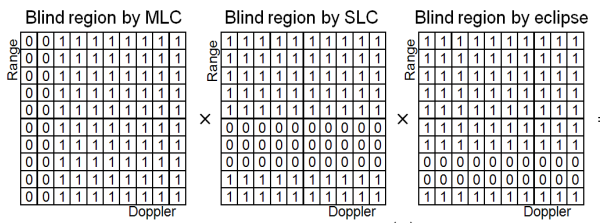

(a)
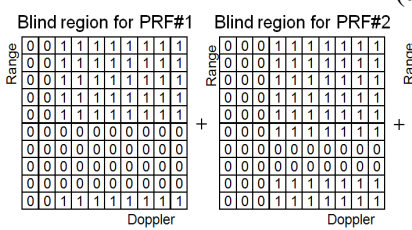

(b)

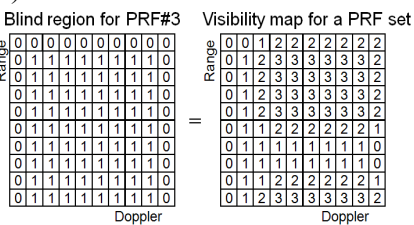

(b)

Fig. 3. Calculation of visibility of a pulse repetition frequency (PRF) set in the region of interest (ROI). (a) Blind region for a PRF (0: blind, 1: visible, $\times$ : array multiply, $=$ : result) and (b) blind region of each PRF and visibility map for a PRF set (visibility=59/100). $\mathrm{MLC}=$ mainlobe clutter, SLC $=$ sidelobe clutter.

determined as the width of the region in which minimum target power is smaller than SLC power, and targets placed within the width are invisible. Next, we determine the blind region of ROI for each blinding factor using the blindness margin in each PRF, and blind regions of PRFs are combined, as shown in Fig. 3. For the MLC, values are assigned to Doppler cells of the ROI according to the blindness margin of the cells in a PRF. If the blindness margin of a cell is negative, a value of 0 is assigned to the cell. If the blindness margin of a cell is positive, a value of 1 is assigned to the cell. A value of 0 represents a blind cell, and a value of 1 represents a clear cell.

The values assigned to Doppler cells of the ROI are extended to the range cells of the ROI. The blind region for MLC is then determined in a PRF. In the cases of SLC and eclipse, values are assigned to the range cells of the ROI according to the blindness margin, and the values are extended to the Doppler cells of the ROI. Blind regions determined by blinding factors are combined by multiplying arrays, as in Fig. 3(a) and then the blind region for a PRF is determined. Blind regions for PRFs are combined by adding arrays as in Fig 3(b); then a visibility map for a PRF set is determined.

In 2-of-3 PRF schedules, if a cell of the ROI is clear in at least two PRFs, a target of the cell can be detected. Therefore, in a visibility map, targets of cells that have values of at least 2 are visible. From the visibility map, the visibility of the 2-of-3 PRF set is calculated by [(visibility of a PRF set)=(number of cells which have value of at least 2)/(total number of cells in ROI)] as in Fig. 3(b). In each battle condition, the values of visibility of the pre-selected PRF sets are calculated by using the method just described for the ROI, and the priorities of the PRF 
sets are determined according to these values of visibility. Finally, the top 3 in the priority list are selected as the triple 2-of-3 PRF set.

\section{Triple 2-of-3 PRF Scheme}

A method of target detection for the triple 2-of-3 PRF schedule is proposed. We first obtain the results of target detection in each PRF set through sequential uses of three 2-of-3 PRF schedules. Some of the three PRF sets may then produce ghost targets in target detection, but the locations of ghost targets within ROI vary according to the PRF set. Secondly, the targets whose locations match at more than two PRF sets are admitted as the true targets from among the detected targets in PRF sets. That is, for the targets detected from three PRF sets, the true targets are confirmed by applying the logic of the 2-of-3 scheme to the triple PRF set, and the ghost targets are eliminated.

\section{Test Simulations and Results}

We showed the effectiveness of the real-time search method of triple 2-of-3 PRF sets by applying the proposed search method to simulated data for traces of an aircraft and ROI (traces of platform and target). That is, the positions of the platform and ROI continuously change in our simulation environment. First, we preselected 762 PRF sets (which satisfied decodability constraints) from ${ }_{N} \mathrm{C}_{3}$ PRF sets. Then, in the location of the traces of an aircraft and ROI (where the main beam looks to the center of the ROI), we calculated the priorities of the PRF sets by calculating their visibilities as in Fig. 3(b). The simulation result is shown in Fig. 4-this result is one of the results for traces of an aircraft and ROI, where the horizontal axis represents the PRF sets sorted by their priorities and the vertical axis represents the true value of visibility for a PRF set, where true visibilities were calculated using the range-Doppler clutter map of each PRF [2].

As shown in the example in Fig. 4, the real-time search method of PRF sets operated effectively. PRF sets were selected in less than $1 \mathrm{~ms}$ for a ROI of range width $3 \mathrm{~km}$ and Doppler width $20 \mathrm{kHz}$ (using Intel $1 \mathrm{GHz} \mathrm{CPU}$ ). The 4-of-9 scheme transmits 9 PRFs and resolves ambiguities using target detections in more than 4 PRFs of 9 PRFs. Our triple 2-of-3 scheme transmits 9 PRFs (sequence of three 2-of-3 PRF schedules). Each 2-of-3 PRF schedule resolves ambiguities using target detections in more than 2-of-3 PRFs, and targets whose locations match at more

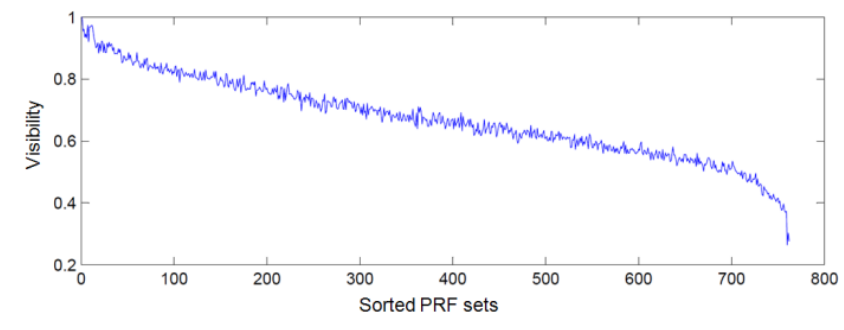

Fig. 4. Visibilities of pulse repetition frequency (PRF) sets sorted by proposed search method in a location of traces of aircraft and region of interest.

than two of three PRF sets are admitted as the true targets (that is, the triple 2-of-3 scheme uses target detections in more than 4 PRFs). Therefore, the triple 2-of-3 PRF schedule was comparable with the 4-of-9 PRF schedule, but the current real-time searching of PRF sets for the 4-of-9 PRF schedule is impossible. In a thousand experiments, the two PRF schedules (triple 2-of-3 and 4-of-9) showed the same performance in target detection and elimination of ghost targets.

\section{Conclusion}

A real-time method that searches optimal or sub-optimal PRF sets according to momentary battle situations was presented for medium PRF pulsed-Doppler airborne radars. An effective method of real-time selection of PRF sets for 2-of-3 PRF scheme was developed and the triple 2-of-3 PRF scheme was proposed for elimination of ghost targets. This proposed method shows good performance and operates in real time.

\section{References}

[1] A. M. Kinghorn and N. K. Williams, "The decodability of multiple-PRF radar waveforms," in Proceedings of the IEE Radar Conference, Edinburgh, UK, 1997, pp. 544-547.

[2] C. M. Alabaster, E. J. Hughes, and J. H. Matthew, "Medium PRF radar PRF selection using evolutionary algorithms," IEEE Transactions on Aerospace and Electronic Systems, vol. 39, no. 3, pp. 990-1001, Jul. 2003.

[3] E. Hughes and M. Lewis, "Advanced detection and tracking in medium PRF radar," in Proceedings of the 4th Electro Magnetic Remote Sensing (EMRS) Defence Technology Centre (DTC) Technical Conference, Edinburgh, UK, 2007. 